\title{
ESTUDIOS Y MIRADAS PARA PENSAR EL FUNCIONAMIENTO DE LA EDUCACIÓN UNIVERSITARIA EN EL CONTEXTO CONTEMPORÁNEO
}

\author{
Frank Casas ${ }^{1}$
}

\section{RESUMEN}

El sistema universitario está en medio de un acelerado proceso de cambio. El inicio del contexto contemporáneo no solo ha provocado reconfiguraciones en las estructuras socioeconómicas y políticas que lo rodean, sino también en la concepción de su propio sentido. Este ensayo sostiene que el proceso de transformación del sistema universitario puede entenderse mejor como relaciones e interacciones, que se presentan mediante luchas y contradicciones que le otorgan un nuevo sentido a la función educativa de la universidad. Su sentido ha cambiado: desde las grandes estructuras políticas y económicas que dirigieron su dinámica y posición en tiempos históricos, por otro relacional, desde la vida cotidiana, donde los actores hacen de la universidad un sistema independiente.

Palabras clave: capitalismo y universidad, Estado y universidad, transformación universitaria, sistema universitario

\section{RESEARCH AND PERSPECTIVES ABOUT THE PERFORMANCE OF HIGHER EDUCATION SYSTEMS IN CONTEMPORARY CONTEXT}

\begin{abstract}
The university system is in the midst of an accelerated process of change. The beginning of the contemporary context has not only caused reconfigurations in the economic and political structures that surround their system but also in the design of their own sense. This essay argues that the transformation of the university system can be best understood as relationships and interactions. These relationships are presented through struggles and contradictions that give a new meaning to the educational function of the university. The direction of the university has changed: from a sense of major political and economic structures directing their dynamics and position in historical times, to other relational sense, from everyday life, where actors make the university an independent system.
\end{abstract}

Keywords: capitalism and university, state and university, university system; university transformation

1 Docente e investigador en la Universidad de San Martín de Porres, Lima. Perú. Contacto: casas.frank@pucp.pe 


\section{ESTUDIOS Y MIRADAS PARA PENSAR EL FUNCIONAMIENTO DE LA EDUCACIÓN \\ UNIVERSITARIA EN EL CONTEXTO \\ CONTEMPORÁNEO}

\section{Introducción}

En el contexto contemporáneo, la universidad ha atravesado un complejo proceso de reconfiguración y adaptación en la formación de su propio sistema. Su historia la presenta como "un instrumento en búsqueda de una verdad traducida en el conocimiento"; como "una institución de sabiduría"; como "un arma poderosa para implantar y sostener posiciones verticales, regímenes y revoluciones ideológicas". Estas reflexiones demuestran la dispersión de su campo de estudio; así como la complejidad de su comprensión.

Para la construcción de este ensayo se ha explorado en la bibliografía especializada e indexada de los "Higher Education Studies", así como de literatura de divulgación de las ciencias sociales para acotar citas referenciales. Esto nos ha permitido estructurar un marco de discusión amplio y diverso, necesario al momento de explorar entornos cambiantes como los del sistema universitario. Reflexionar desde su campo académico nos ha permitido reconocer los alcances y límites de su fenomenología, básicos para comprender su proceso de transformación en tanto origen y función para con la sociedad.

\section{Procesos del sistema universitario desde la visión del Estado contemporáneo}

El estudio de los sistemas de educación superior universitaria tiene diversos enfoques de tratamiento y análisis, uno de ellos es la mirada estructural de sus procesos. Por ende, un examen sobre el proceso puede esquematizar al sistema universitario como movimiento estructural que surge como una forma de pensar el poder. Así, la discusión se concentra en torno a los fenómenos 
sociales y políticos del saber: ¿cómo aparece?, ¿en qué contexto?, ¿hacia dónde se dirige?

En la redefinición de las universidades contemporáneas, una de las características que pautan el surgimiento (y por consiguiente la identificación) de la sociedad postindustrial es la condición de saber. A partir de esta, las estructuras sociales y políticas se observan por separado. El saber técnico es el papel central que jugaría el conocimiento en esta sociedad (Neave, 2007; Clark, 1983). Queda claro que en el postindustrialismo, el conocimiento, la sabiduría y el saber no dejan de relacionarse con el crecimiento económico. La visión "tecnicista" parece marcar la pauta de una mirada sobre la realidad, no solo en el sistema universitario, sino en las estructuras del Estado para ejecutar su visión económica (Magalháes, Amaral, 2009; Olsen, 2007). Pero al mismo tiempo, los nuevos aires de liberalización no permiten que reflote una sociedad líquida de ocio y consumo (Scott, 1995). En palabras de Touraine (1969, pp. 7-8), "estamos en una época de constantes reconfiguraciones".

La universidad contemporánea aparece en una relación compleja; cambiante, dinámica y caótica. Surge el reconocimiento de nuevos derechos y deberes, en un contexto de luchas, ideologías y represiones que devienen en conflictos contantes. "La relación entre la estructura social y el poder político se convierte por eso mismo en uno de los problemas claves del poder en una sociedad postindustrial" (Bell 1989: 29). De esta manera se reconfiguran nuevas maneras de pensar, vivir y utilizar la universidad.

Existe un consenso entre los autores que han tratado de comprender el sistema universitario en la postindustrialización, al señalar que el papel determinado por la universidad se construye sobre el significado cambiante de la educación y la idea emergente de la educación superior (Barnett, 1994). Como el manejo del conocimiento profesional es, por excelencia, instrumento de la educación superior (Clark, 1983), gran parte de este cambio debe ser buscado en el papel cambiante del conocimiento en las sociedades. 
"Como ya lo mencionamos, las universidades, junto con la Iglesia Católica, se encuentran entre las más viejas instituciones de existencia continuada en la sociedad moderna. Quizá sea poco razonable suponer que instituciones tan estables -por no decir inerciales- puedan ser reformadas o transformadas desde adentro." (Arocena, 2001, p. 311).

Ambas miradas parecen marcar una pauta discursiva para la discusión acerca del papel de la universidad en la formación de conocimiento para la sociedad. Empero, la determinación economicista de la función de la universidad (Allen y Van der Velden, 2005) deja inmensos vacíos cuando se desea discutir sobre la generación del valor del conocimiento. Por ello, es importante entender cuáles son las perspectivas para el trabajo tecnificado, y cuánto y cómo puede ajustarse la intelectualidad al sistema de producción capitalista.

El capital humano parece ser la clave para discutir y comprender este elemento híbrido que denominamos conocimiento técnico (Carneiro y Heckman, 2003). Este no necesariamente se aleja del intelectualismo de la universidad clásica ni de la tecnificación terciaria, sino que el propio sistema educativo lo configura para autoorganizarse, trayendo como resultado la imperiosa necesidad de certificación, calidad educativa y trabajo por competencias (Sternberg, 1997; Hymes, 1996). La disminución del interés de las investigaciones en ciertos campos del saber (estudios en ciencias sociales y humanidades) son costos que la sociedad debe demandar para sostener su crecimiento (Giusti y Patrón, 2010).

Elementos como la competitividad y la innovación configuran nuevos aspectos para visionar el interés por la certificación y la calidad educativa, tanto en relaciones sociales como por el estatus de procedencia universitario y, finalmente, en el rendimiento académico. En todo caso, determinar la fortaleza de las redes sociales que se tejen sobre el capital humano no se puede determinar con una visión optimista de la competencia mercantil por certificar, sino que necesariamente debemos rastrear la visión del Estado para imponer el direccionamiento de sus políticas económicas. Ante ello, el enfoque estructuralista localiza discursos políticos sobre las redes y posición 
del Estado en un contexto globalizado y de libre mercado (Nelson, 1993). El neoliberalismo trae cambios traducidos en nuevas formas de paradigma de gestión perteneciente al movimiento llamado Nueva Gestión Pública (NGP). Esta sirvió como elemento catalizador para proponer la mercadización de todos los sectores estratégicos del Estado. El impulso de la autonomía académica y empresarial y la reorganización del sistema de gobierno son la piedra angular de las reformas de dicho discurso para el incentivo de las "universidadesmercado" (Moreira, 2008: 125).

Sin embargo, surge un problema ontológico, decir que los sistemas de educación superior necesitan de autonomía académica y económica es una estrategia discursiva que tiene el remedio para una supuesta resistencia, es decir, para la redefinición de que las universidades públicas, en concordancia con lo privado, necesitan ser organismos autoadministrados. Bajo la influencia de la NGP, la estrategia del Estado se enfoca en reformar la dirección política y transferir la gobernabilidad de la educación como supervisión estatal a la junta directiva. La autonomía es el logro final de la evaluación del Estado y la etapa final en su transformación de "Estado guardián" a "Estado observador y poco regulador" (Neave, 2007).

Las suposiciones sobre el papel del conocimiento dan lugar a nuevos enfoques de redes de política (Meek y Wood, 1997) que han vuelto a colocar a la educación superior en el contexto de lo político. Esta concepción no solo genera cambios en las prioridades estratégicas de las universidades, sino también en el gobierno y los cambios organizacionales. Para la sociedad el mercado laboral terciario es el indicador central para pensar sobre el rol de la universidad. Esta puede articular a las otras miradas en función de la expansión y demanda, ubicando al sistema como un símbolo de acceso a un mundo de posibilidades económicas, políticas y sociales sobre una base discursiva de libre mercado. Lo universitario se articularía como tal cuando hace referencia a juegos instrumentales para oportunidades laborales competitivas y como redes políticas para el prestigio social.

En ese contexto surge un problema mayor: la universidad tecnicista no logra solucionar la dificultad del acceso económico en 
los estratos medios y emergentes. En la contemporaneidad los marcos instrumentales de la educación universitaria pueden verse trastocados cuando sobresale una matriz compleja y abstracta de sobreponer la vocación profesional, sin que esta se relacione totalmente con el campo intelectual del conocimiento o con el terciario. "La 'formación vocacional' es claramente un fenómeno de larga duración que, si bien ahora vuelve a ser una fuerza central en el establecimiento de los sistemas europeos, tiene un considerable peso histórico" (Neave, 2001, p. 125).

La universidad se ha reconfigurado como una institución pragmática sobre estructuras sociales cada vez más fragmentadas e interdisciplinarias. Es el correlato con las estructuras sociales y sus prácticas concretas las que marcan la fragmentación de la universidad. El tema central es que bajo esta estructura de fraccionamiento discursivo de la universidad, la política y la fragmentación social en el terreno del mundo de la vida, el sistema universitario atraviesa modificaciones en el ejercicio de las prácticas educativas, y en la función de sus instituciones. En el contexto contemporáneo las políticas públicas educativas son las que ejecutan dichas funciones.

\section{Debates sobre la función de las políticas públicas educativas}

La reflexión sobre el estudio de las políticas como función de educación universitaria nos exige determinar enfoques de tratamiento y análisis. Una de ellas es la mirada antagónica de su interacción sistémica entre la sociedad y el Estado. Una mirada sobre su papel funcional puede esquematizar al sistema universitario como elemento prioritario, o como razón de política de Estado. ¿Cómo puede el Estado articular las estructuras del sistema universitario sobre entornos complejos?

La política pública universitaria como función educativa desde el Estado desarrolla una articulación estructural que determinaría el mejor modelo formativo integral. Esta mirada se ejemplifica con las observaciones de estructuras universitarias, como por ejemplo, la admiración por los sistemas norteamericanos, asiáticos y/o europeos, 
donde, pese a la dicotomía ciencia y tecnología, se ha configurado un sistema adaptativo a sus necesidades. Esto demostraría la eficacia de las políticas nacionales y sectoriales de cada jurisdicción de los continentes mencionados. Ergo, el poder del Estado estaría ejerciendo una presión de direccionamiento, en sus reformas y crisis, sobre la universidad.

Siguiendo a Bell (1989), las demandas sociales, en el contexto contemporáneo, requieren de un vínculo entre el saber en la toma de decisiones políticas, lo que implica la burocratización del quehacer intelectual (la universidad cimienta su estructura y sus procedimientos) y la legitimación de un saber técnico que es necesario para promover y defender determinados derechos ciudadanos (donde el aparato administrativo de la universidad ejecuta los insumos económico y financieros; mientras la asamblea universitaria discute sobre la gobernabilidad del sistema).

"Mucho de lo que se escribe hoy sobre el Estado evaluador gira alrededor de su instrumentalizado, esto es, la dimensión técnica de los sistemas institucionales de administración, sus repercusiones en la productividad académica (ya sea en el área del establecimiento del cuerpo docente, del departamento o en el nivel individual), sobre sus consecuencias en el sector del poder, la influencia y la jerarquía, reservada o acordada de mala gana al personal académico o recientemente concentrada en las pujantes filas de 'gerentes'; sobre los costos unitarios y sobre los instrumentos de una nueva inspección: indicadores de rendimiento, índices de calidad, fijación de estándares mediante "cotas de referencia"' (Neave, 2001, p. 216).

El elemento paradójico de esta perspectiva estatal es la autonomía universitaria (en su conjunto, ya no solo de cátedra), como filosofía de verdad para generar conocimiento. La autonomía universitaria se establece como una conquista de la propia gobernabilidad universitaria frente a los poderes locales, del Estado y de las instituciones no estatales como la Iglesia. Ejemplo de aquello es el paso histórico de la "universitas magistrorum" de la Universidad de París a la visión "humboldtiana" de la Universidad de Berlín, hasta finalmente llegar a la primera reforma de Córdoba (Burga, 2008). 
Si bien el Estado mantiene un eje preponderante para establecer políticas de lineamiento, incluso sobre su incapacidad y decisión para regular la relación entre universidad y Estado, no proporciona ninguna imagen de su poder sobre la sociedad. Para Perkin (2006) la universidad como sistema pertenece a las estructuras de la sociedad, por lo cual mantiene su autonomía relativa frente al Estado, que, como lo vemos hoy en día, no ha podido utilizar para el desarrollo de sus fines. Ahora bien, ¿cómo se estructura esta relación relativamente autónoma?

Una mirada que pretende responder esta pregunta está en las reformas de las instituciones, donde grandes procesos sociales y económicos, relacionados con el intelectualismo y el saber, no se gestaron desde la universidad sino fuera de ella; como contraparte, el Estado, asumiendo la gran pérdida de poder sobre las universidades, muchas veces estimuló la formación de grupos y segmentos que pelearon en contra de su autoridad e intereses económicos. El Estado ya no dirige la vida universitaria sino que el sistema tiene el poder de autorrealizarse y dirigir su propio destino (Perkin, 2006).

"Las universidades han sido hasta aquí el refugio secular de los mediocres, la renta de los ignorantes, la hospitalización segura de los inválidos y -lo que es peor aún- el lugar en donde todas las formas de tiranizar y de insensibilizar hallaron la cátedra que las dictara. Las universidades han llegado a ser así fiel reflejo de estas sociedades decadentes que se empeñan en ofrecer el triste espectáculo de una inmovilidad senil. Por eso es que la ciencia frente a estas casas mudas y cerradas, pasa silenciosa o entra mutilada y grotesca al servicio burocrático" (Manifiesto Liminar, 1981).

Esta mirada de la autonomía relativa del Estado frente a la universidad configura la necesidad de que las políticas públicas se traduzcan en toma de decisiones incrementales y técnicas. La mirada sobre el valor público, desde una perspectiva clásica como la de Moore (1998), devuelve a la universidad, pensando en las limitaciones complejas de la realidad, el interés por emprender líneas de acción continuas que beneficien a la sociedad. El valor público obliga a la universidad y al Estado a estar pendientes de las percepciones y 
reacciones de la sociedad para mantener diálogos sobre lo que resulta valioso y lo que hay que repensar. El ser humano, como centro de la política, desmorona las determinaciones economicistas de los sistemas universitarios en contextos de desarrollo capitalista, y mira hacia las nuevas relaciones de poder entre Estado, desarrollo económico y sistema universitario.

\section{Transformación universitaria y relaciones de poder}

Lo que nos queda claro es que los estudios del proceso de transformación del sistema universitario en el mundo no pueden ser analizado solamente desde las fragmentaciones autónomas de las estructuras sociales y de las políticas-ideológicas; por el contrario, lo que vemos es que en el proceso histórico de la universidad se han venido estructurando redes dinámicas de interacción sobre el poder. Estas reconfiguran las prácticas endogámicas del propósito universitario. No es en el nacimiento de las universidades donde se conforma el sistema, sino que este se modela sobre nuevos paradigmas que atraviesan nuevos intereses de grupo: la ecología académica ha cambiado (Trow, 2005).

Este enfoque modifica la visión instrumental por una que configura las nuevas formas de producción y consumo de información en esta fase del capitalismo como proceso hacia la sociedad del conocimiento (Cobo y Moravec, 2011). El conocimiento, por consiguiente, no es un instrumento sino un proceso que se construye rompiendo viejos paradigmas monopólicos del saber.

La mirada educativa sobre el desarrollo económico analiza cómo el conocimiento existente, a menudo, no se introduce en los mercados. Esta perspectiva busca intervenir en la investigación académica y propiciar esfuerzos por innovar en ciertos desarrollos tecnológicos que beneficien y promuevan la competitividad del capital público y privado, local o internacional. El punto estratégico de acción está en la vinculación de la universidad con la industria, por medio de prácticas pedagógicas informales, fuera de las paredes de las casas de estudio. 
Desde la perspectiva de sus funciones, las universidades deberían mantenerse concentradas en la docencia y en la formación de "recursos humanos" con una malla curricular basada en competencias, o bien reproducir la calidad de la "investigación básica", para dejar que el desarrollo tecnológico, en función de lo técnico, y la innovación con fines de elevación de la productividad se concentren en la vida de las personas (Reichert, 2006).

Si algunas categorías de educación (enseñanza, aprendizaje, estudiantes, profesores, clases, etc.) se basan en una función formativa atribuida al conocimiento, la reconfiguración de la educación está provocando una importante remodelación en la función educativa del conocimiento, es decir, su función formativa está siendo reconfigurada por el potencial de las nuevas prácticas de relaciones sociales (Cobo y Moravec, 2011), tanto en el mundo de la economía como en el del trabajo. Según esto, hoy en día la gestión educativa es vista como un producto y como un "aprendizaje por resultados" (Magalhães y Sousa, 2007).

Esto cambia con la mirada constructivista del conocimiento, donde los modos formales de aprendizaje social conviven con las nuevas formas de producir y consumir conocimiento en la "sociedad red" (Castells, 1991). Internet ha acelerado este proceso interrelacionando redes de conocimientos (formales e informales), y donde el acto comunicativo -no tanto el mensaje- construye la complejidad autorreferencial del sistema dinámico universitario.

En tanto, en las estructuras universitarias formales los estudiantes son al mismo tiempo grupos de interés interno, clientes de los servicios educativos y personas envueltas tanto en temas educativos como de mercado de trabajo. Ellos son el centro de interés que busca la empresa, mientras el profesor se reinventa como un "diseñador instruccional" (Cowen, 1996). El sistema -hoy más que nunca- reorienta su visión al ser humano como conocimiento y centro de aprendizaje.

De lo escrito, entendemos que la mirada sobre las nuevas formas de desarrollo capitalista para producir y consumir información 
está provocando alteraciones en el modelo postindustrial y que, a su vez, permite el rompimiento de la institucionalidad monopólica de los centros formales del conocimiento. El desarrollo desde la educación superior universitaria obliga a ubicar una nueva fase del mercado capitalista que, ciertamente, modele nuevas formas de poder descentralizado. ¿Hacia dónde se dirige la universidad hoy?

\section{Conclusiones}

Las redes informales del conocimiento y las formas de poder político y económico de las universidades constituyen un mecanismo de relación que permite conectar a los actores con el sistema. Se trata de entender a la universidad como un sistema complejo de múltiples interacciones. Atrás quedaron las miradas instrumentalistas que determinaban la expansión del sistema universitario sobre discursos ideológicos, luchas y contradicciones de un sistema que debiera situarse en las estructuras sociales o del poder político. Atrás quedó la institucionalización del sistema únicamente como un mecanismo de acceso al mercado laboral. Vale la pena insistir, es la relación entre las formas de producir conocimiento en la sociedad y las estructuras políticas lo que nos interesa comprender.

Este tipo de relación no es entendido únicamente como un proceso de bienes fácticos de poder, sino también como un intercambio de bienes simbólicos (Bourdieu, 1997), dentro de un contexto cambiante y turbulento. Dichos bienes poseen una carga económica, política, y una nueva disposición en la construcción de valores sociales. Todas estas son alianzas, redes y complicidades, creando formas locales de reciprocidad o negociaciones determinadas. Hemos trascendido de un enfoque metodológico macro del sistema universitario hacia sus microestructuras; es en la realidad etnográfica de la universidad donde podemos comprender los juegos de poder, las transgresiones y su transformación.

"Estas múltiples alianzas son parte de las formas de ejercicio de poder que nos hacen entender lo político de otra forma. Se trata de descentrar a este de las estructuras del Estado, de llevarlo al terreno de la cultura, de nuestras actividades cotidianas y, por ende, 
empezar a estudiarla en el terreno de la performance de los actores. Esto flexibiliza su posición, remueve sus cimientos y muestra sus partes móviles: aquellas que se transforman con el movimiento de los sujetos" (Mujica, 2010, p. 11).

Reflexionar sobre esta nueva forma de pensar la universidad es fundamental para ubicar su proceso de transformación desde adentro. Lo que nos toca es investigar sobre las estructuras de la universidad desde la vida cotidiana, desde las redes de sus actores, desde las relaciones del poder político y el educativo. El fenómeno de la expansión del sistema universitario en el mundo es un buen ejemplo. Su aparición vertiginosa no solo se debe a estructuras hegemónicas, o como fruto de un mercado formal destinado a la tecnocracia, sino que también constituye conflictos, disputas, negociaciones, movimiento del poder, reinvención del conocimiento, que transforman el sistema desde adentro, no como anécdotas de la vivencia universitaria, sino como entidades constituyentes de su estructura. La universidad debe ser vista como una entidad de comunicación, de redes y de relaciones. Hoy la universidad se "autoperforma" ya no puede verse como una variable dependiente.

\section{Referencias bibliográficas}

Allen, J. y Van Der Velden, R. (Eds.) (2005). The flexible professional in the knowledge society: Conceptual framework of the REFLEX Project. Research Centre for Education and the Labour Market. Netherlands, Maastricht University.

Arocena R. y Sutz, J. (2001). La Universidad Latinoamericana del Futuro. Tendencias - Escenarios - Alternativas. Universidad de la República Oriental del Uruguay. Colección UDUAL 11.

Barnett, R. (1994). The Idea of Higher Education (Society for Research into Higher Education). Buckingham, SRHE and Open University.

Bell, D. Industria cultural y sociedad de masas. Caracas, Monte Ávila, 1974.

Burga, M. (2008). La reforma silenciosa. Descentralización, desarrollo y universidad regional. Lima: Red para el Desarrollo de las Ciencias Sociales en el Perú.

Carneiro, P. y Heckman, J. (2003). Human Capital Policy. IZA Documento de trabajo.$^{\circ} 821$. 
Castells, M. (2001). La Era de la Información. El poder de la identidad. Volumen 2. México Distrito Federal, Siglo XXI Editores.

Clark, B. (1983). The Higher Education System. Academic Organization in CrossNational Perspective. Berkeley. University of California Press.

Cobo, C. y Moravec, J. (2011). Aprendizaje invisible. Hacia una nueva ecología de la educación. Cataluña. Col-lecció Transmedia XXI. Laboratori de Mitjans Interactius.

Cowen, R. (1996). Perfomativity, Post-modernity and the University. Comparative Education. Volumen 32, n. ${ }^{\circ}$ 2, pp. 245-258.

Giusti, M. y Patrón, P. (2010). El futuro de las humanidades. Las humanidades del futuro. Lima. Fondo Editorial PUCP.

Hymes, D.H. (1996). Acerca de la competencia comunicativa. Traducción de Juan Gómez Berna. Forma y función. Santa Fe de Bogotá, n. 9, pp. 13-37.

Magalhães, A. y Amaral, A. (2009). Mapping out Discourses on Higher Education Governance. En Huisman, Jeroen (editor) International Perspectives on the Governance of Higher Education. Alternative Frameworks for Coordination. United Kingdom: Taylor \& Francis.

Manifiesto Liminar de la Reforma Universitaria (1981). La Juventud Argentina de Córdoba a los Hombres Libres de Sudamérica. Argentina.

Meek, L. y Wood, F. (1997). The market as a new steering strategy for Australian higher education. Higher Education Policy. Australia, volume 10 , n. ${ }^{\circ} 3 / 4$, pp. 253-274.

Moore, M. (1998). Gestión estratégica y creación de valor en el sector público, Barcelona, Paidós.

Moreira, V. (2008). The legal status of higher education. En Amaral, Alberto Politicas de Ensino Superior - Quatro Temas em Debate. Lisbon: Conselho Nacional de Educação.

Mujica, J. (2010). "Hacia una etnografía del Parlamento peruano". Documento de trabajo. Lima, PUCP.

Neave, G. (2007). From Guardian to Overseer: Trends in institutional Autonomy, Governance and Leadership. Lisbon. The CNE Conference on Institutional Autonomy, Governance and Leadership.

Neave, G. (2001). Educación Superior: historia y política. Estudios comparativos sobre la universidad contemporánea. Barcelona, Gedisa.

Nelson, R. (1993). National Innovation Systems: A Comparative Analysis. Oxford: Oxford University Press. 
Olsen, J. (2007). The Institutional Dynamics of the European University. En Peter Maassen y Johan Olsen (editores). University Dynamics and European Integration. Netherlands: Springer.

Perkin, H. (2006). History of Universities. En International Handbook of Higher Education. United States: Springer International Handbooks of Education. Volume 18, n. ${ }^{\circ}$ 1, pp. 159-205.

Reichert, S. (2006). The rise of knowledge regions: Emerging opportunities and challenges for universities. Bruselas, EUA Publications.

Scott, P. (1995). The Meanings of Mass Higher Education. Buckingham: Society for Research into Higher Education and Open University.

Sternberg, R. (1997). Successful intelligence. New York: Simon \& Schuster.

Touraine, A. (1969). La sociedad post-industrial. Barcelona: Ariel.

Trow, M. (2005). Reflections on the Transition from Elite to Mass to Universal Access: Forms and Phases of Higher Education in Modern Societies since WWII. En International Handbook of Higher Education. Part One: Global Themes and Contemporary Challenges. Springer International Handbooks of Education.

Recibido: 22/05/2012

Aceptado: 27/09/2012 